\title{
RATIONALE FOR NASA PLANETARY \\ EXPLORATION PROGRAM
}

\author{
I. RASOOL, D. HERMAN, D. KERRISK, and W. BR UNK \\ NASA Headquarters, Washington, D.C., U.S.A.
}

\begin{abstract}
NASA program of future solar system exploration in the years 1973-1990 is presented.
\end{abstract}
\section{Background}

The guiding philosophy in the formulation of the NASA Planetary Program is the concept of balanced exploration. This concept involves a strategy of broad exploration of the entire solar system, rather than concentration on the detailed exploration of one or two planets. Thus, within appropriate levels of resources and technology, the NASA program seeks a balance between the expansion of knowledge by the inclusion of new targets, and the refinement of knowledge obtained on previously investigated targets. It also favors a reasoned balance between expenditures on immediate missions and investment in the development of the technology which make future missions possible.

In formulating its solar system exploration program, a major consideration has been the development of a progressive understanding of each target, so that proposed missions may build on the results and understanding generated by their predecessors. Generally, the sequence of proposed exploration goes as shown in Figure 1, with Earth-based observations being first augmented by flyby missions, then considerably improved by orbiter missions. Details of planetary atmospheres, composition, structure, and dynamics are then investigated by atmospheric probes, followed by landers to study geology, and, where deemed appropriate, biology. Finally, sample return missions to allow Earth-based analysis are considered.

A second consideration in formulating the Planetary Program has been the availability of spacecraft technology. The program has been geared to an evolutionary spacecraft development, with each mission building on the technology of its predecessors. This minimizes step-function changes in the level of technology required for any spacecraft. Where such step-function changes are required (as, for example, in the introduction of RTG power sources) research and technology development programs have been formulated to bring the new technology items to a state of flight readiness, prior to committing the mission.

A third consideration in formulating the program has been the availability of the propulsive capability required for each mission. Requirements are set by the laws of celestial mechanics, mission objectives, target and year of opportunity. Against these requirements we have the finite capabilities of vehicles currently being developed. The phasing of the program must be consistent with matching the mission requirements to launch vehicle availability. 


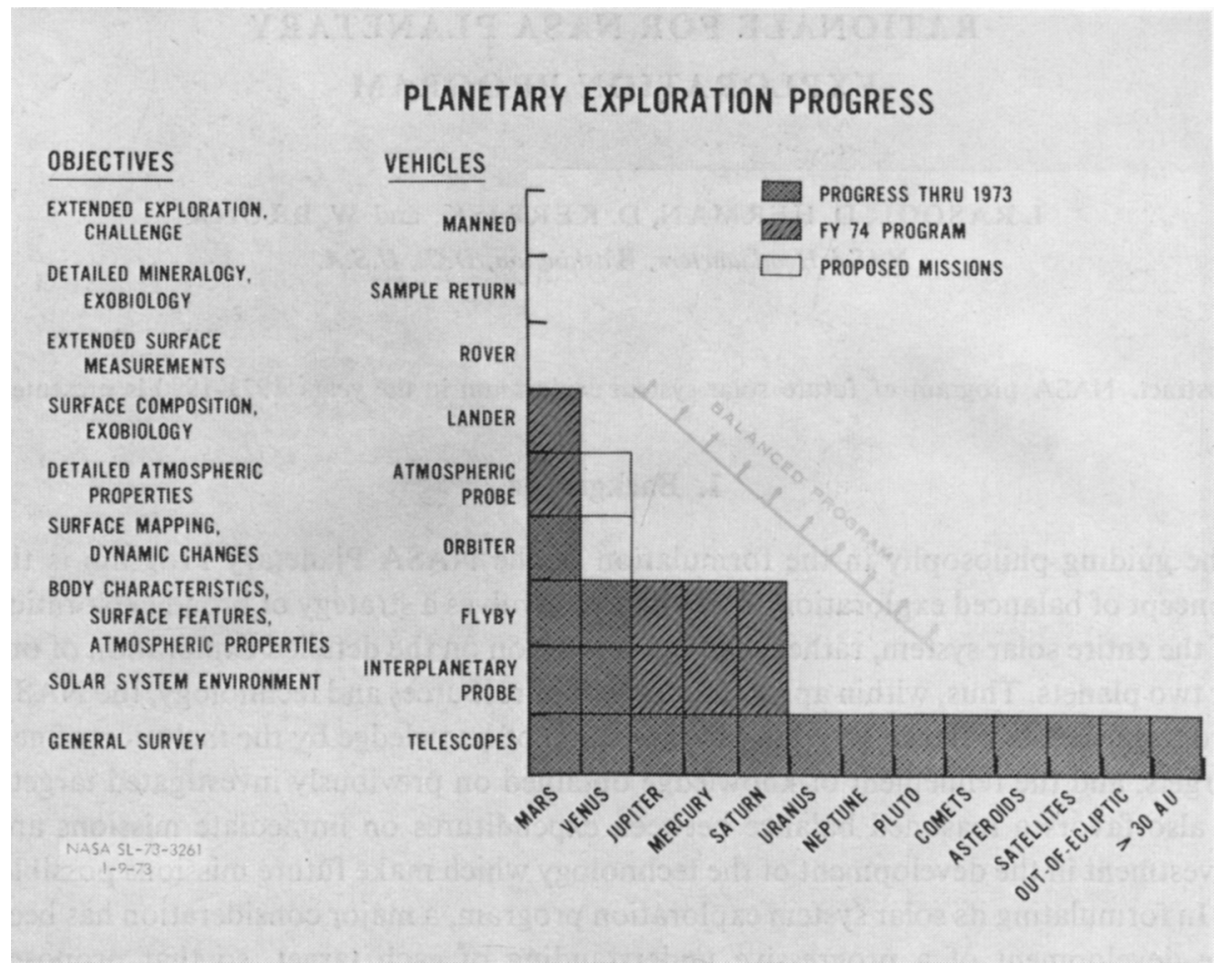

Fig. 1. Planetary exploration progress.

Finally, the program must be formulated within the bounds of realistic fiscal constraints, taking into account not only the mission costs, but also the costs and cost phasing of new technology and enhanced launch vehicle capability development.

The operation of these constraints can be observed in the development of the Planetary Program to date. Planetary exploration started with the mission of Mariner 2 to Venus in 1962, with spacecraft and launch vehicle based on Ranger technology. The introduction of the Atlas/Centaur launch vehicle allowed the Mariner 4, 5, 6, and 7 missions to Mars and Venus, with increasingly sophisticated spacecraft subsystems. Development of liquid retro-propulsion capability preceded the Mariner 9 MarsOrbit mission and the development of RTG power sources allowed the extension of our exploration to the outer planets with Pioneer 10 and 11 . Increasingly sophisticated trajectory, guidance, navigation, and orbit determination software programs have made the use of planetary gravity assisted swingbys a feasible means of extending launch vehicle capability to targets that are outside their capability for direct flights; this will be used for the first time later this year in the Mariner Venus/Mercury Program to extend the Atlas/Centaur capability to provide a flyby of Mercury.

The introduction of the Titan/Centaur launch vehicle in 1974 will provide another step function increase in the NASA exploration capability. This will be used for the 


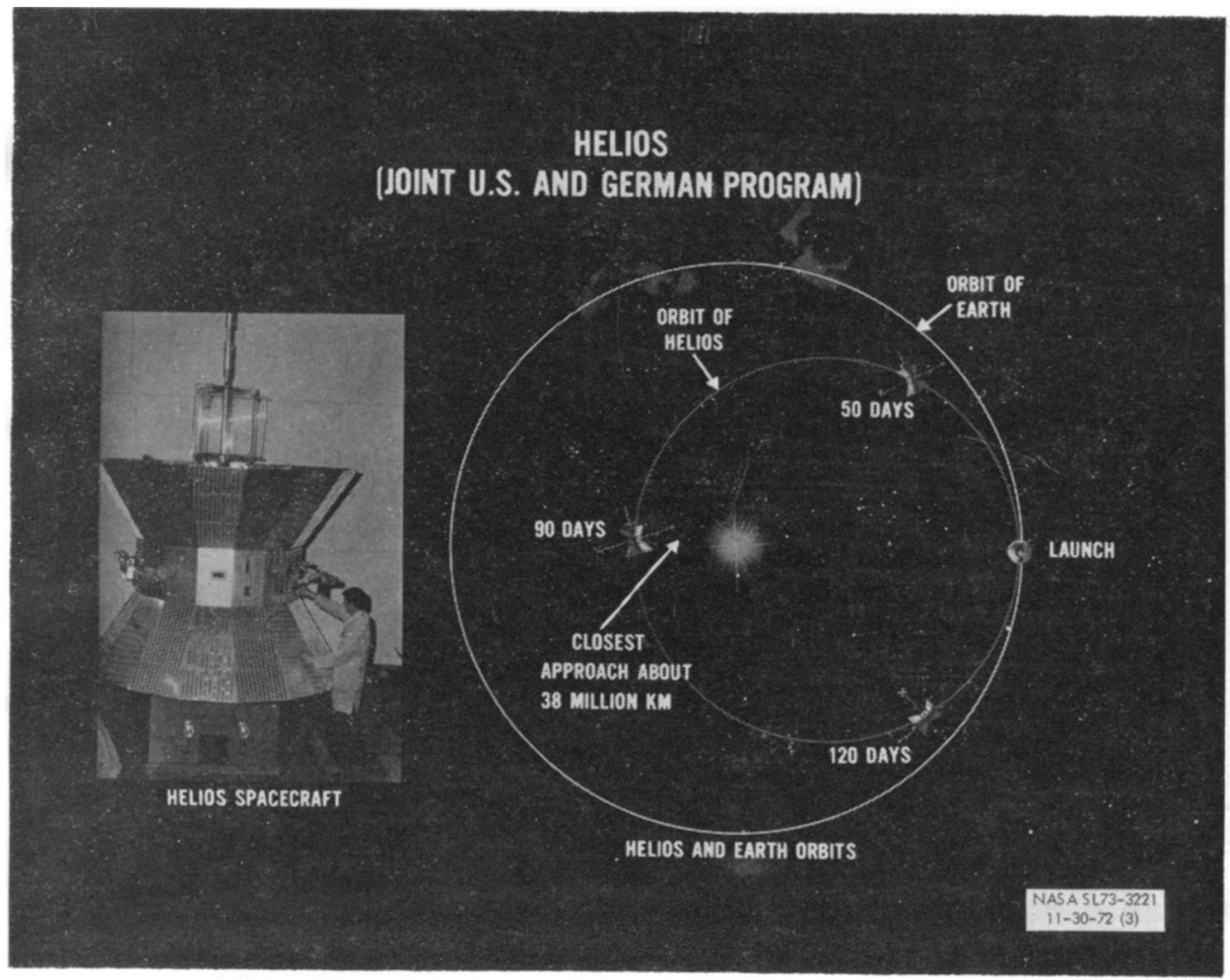

Fig. 2. Helios mission.

first time to obtain a close solar probe in the joint U.S./West German Helios Program (Figure 2) and then will in 1975 send Viking to Mars to perform NASA's first planetary soft landing. In 1977, again taking advantage of planetary gravity assist to enhance the launch vehicle capability, NASA will expand its exploration with the MJS flyby of Jupiter and Saturn providing the first close pictures of Jupiter, Saturn, Saturn's Rings, and the satellite Titan.

It is in the context of this background of completed and ongoing programs that the Planetary Mission Model, defining potential future programs has been constructed.

\section{The Planetary Mission Model}

The present Planetary Mission Model (Figure 3) is based on a continuation of the balanced exploration philosophy, tempered by a conception of the realities of technology availability and fiscal constraints. It must be emphasized that the mission model contains projections of typical missions that might occur in a future time frame and, as such, is subject to continual modification and updating. Iterations and modifications will be made to reflect changes necessitated by budgetary or programmatic considerations; to incorporate results from advanced mission studies; and to 


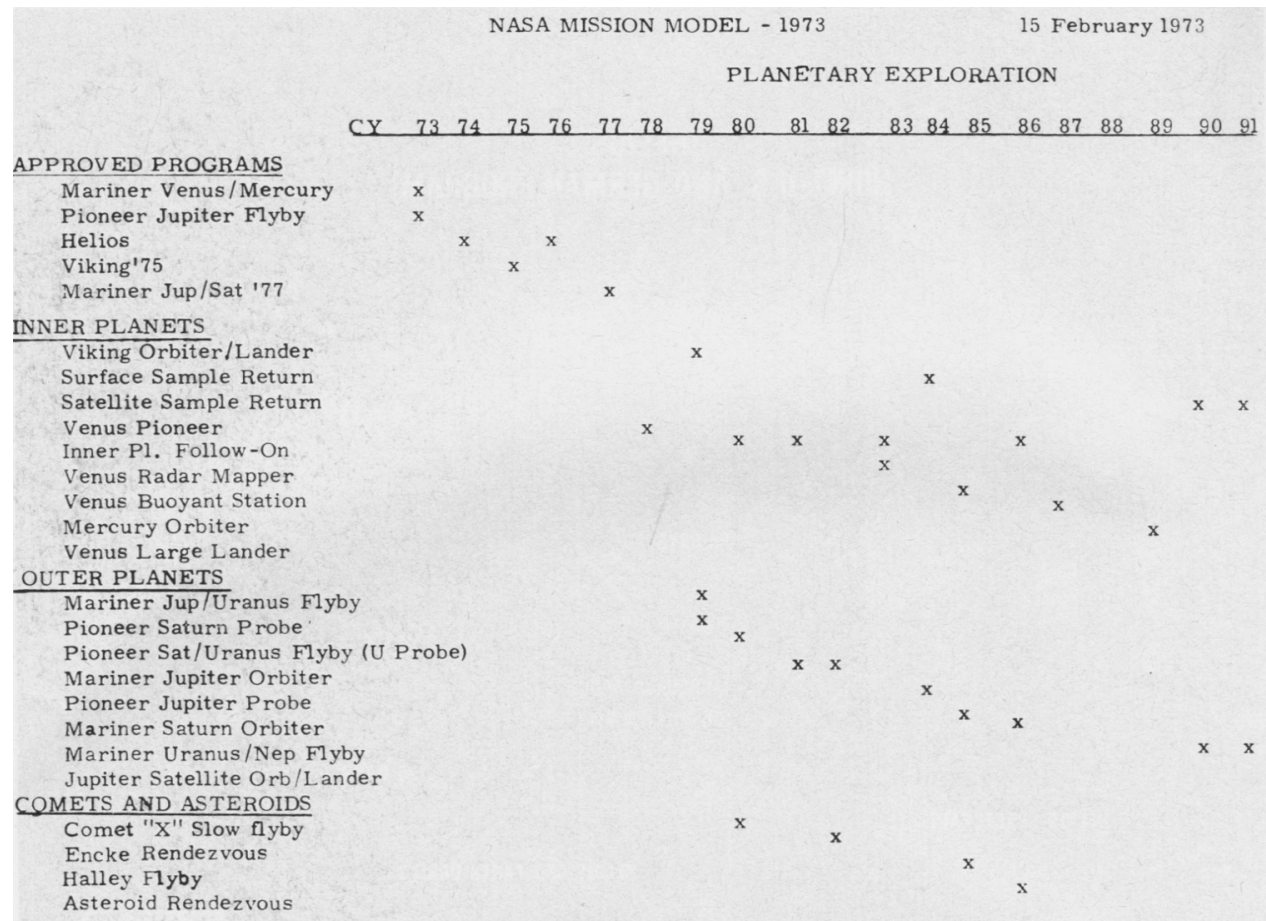

Fig. 3. Planetary mission model.

incorporate results from previous missions. The mission model envisions the continued use of existing spacecraft technology (i.e. Mariner, Viking, and Pioneer) with only evolutionary changes, but with the introduction of atmospheric probe technology in the late 70's. It envisions the continued use of the Titan/Centaur vehicle through 1980, with the introduction of solar-electric propulsion (SEP) capability about 1979. Beyond 1980, shuttle capability is assumed, although all of the missions through 1985 are also compatible with Titan/Centaur or Titan/Centaur/SEP.

The sequencing and phasing of the missions incorporates the recommendations of the scientific community. A brief explanation of the rationale behind the sequences at each target will be presented.

\subsection{Mercury}

The very high energy requirements for reaching Mercury were beyond the capability of the Atlas/Centaur until the gravity assist mode became practical. Using a gravity assist at Venus, the MVM'73 spacecraft will obtain our first exploratory information on Mercury early in 1974. This mission is depicted in Figure 4. The spacecraft has a complement of seven experiments including a television camera to obtain images of the planetary surface. Other experiments will provide data on Mercury's physical properties, surface temperature, atmospheric composition and particle and fields environment. Orbiting the planet however will require the use of multiple Venus 


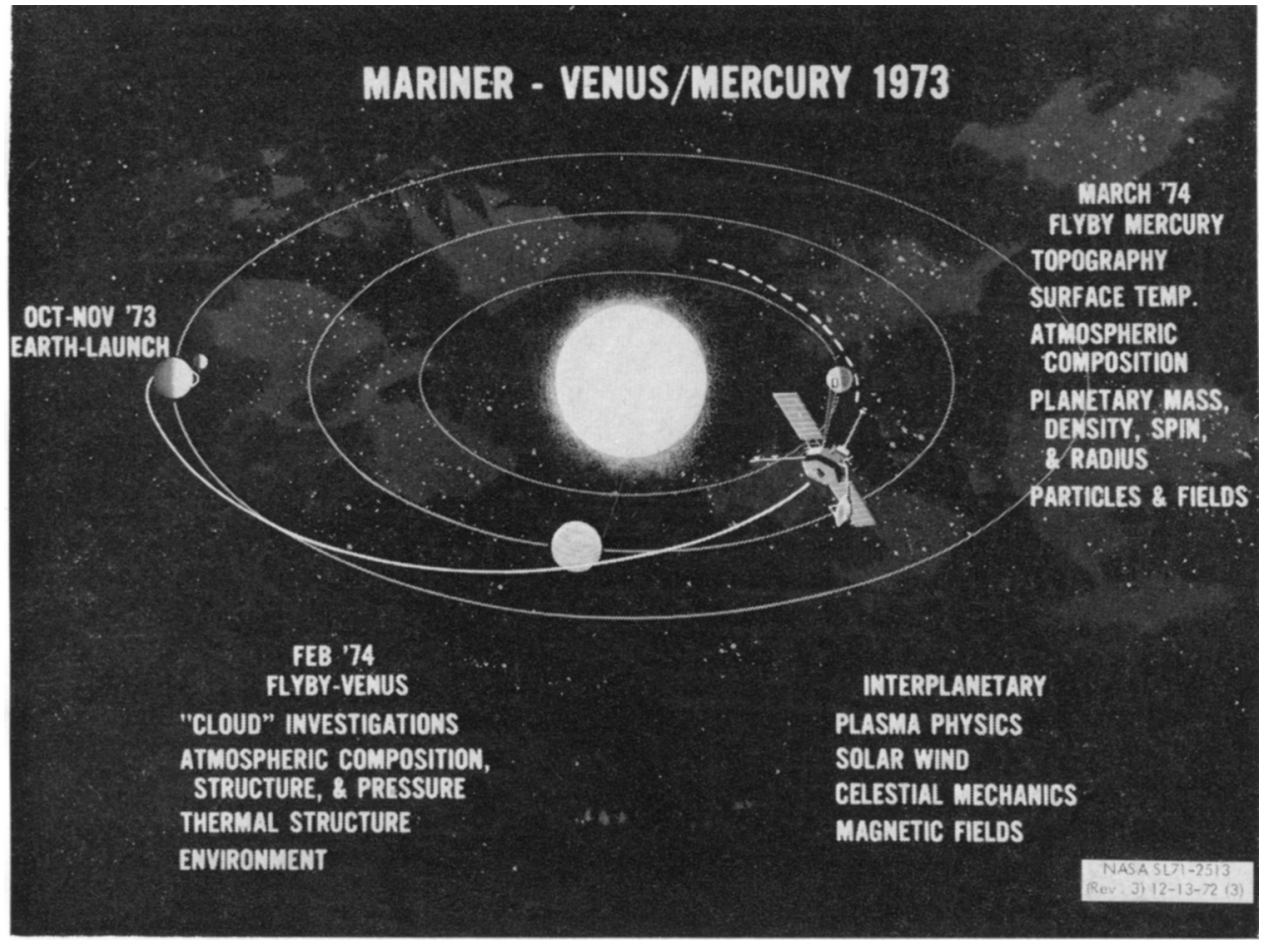

Fig. 4. Mariner-Venus/Mercury mission.

swingbys with very restricted launch opportunities or an advanced SEP system; currently it is estimated this capability will be available in the mid 80's. Before such a mission can be planned, the data from MVM'73 must be thoroughly assessed. Pending this assessment and based on estimated technology availability and funding constraints, a Mercury Orbiter mission is tentatively scheduled for 1987.

\subsection{VENUS}

Venus, as Earth's nearest neighbour and the planet most closely resembling Earth in gross physical characteristics, is an object of considerable interest. The MVM'73 spacecraft will obtain data on the planet's atmospheric properties including composition and structure as well as 'cloud' investigations. It will image the planet, and imaging will considerably enhance present Earth-based resolution to the level shown in Figure 5. Because of the dense cloud cover, however, it cannot be visually mapped. Also the apparent extreme hostility of its surface environment, as deduced by the Mariner 2 and 5 flyby experiments and the Russian Venera spacecraft, means that survivable landing vehicles will require considerable advance in packaging, thermal control, and corrosion resistance technologies.

From one aspect, however, the hostility of the Venusian atmosphere is an aid to scientific investigation. There are strong cosmological arguments that the very dense 


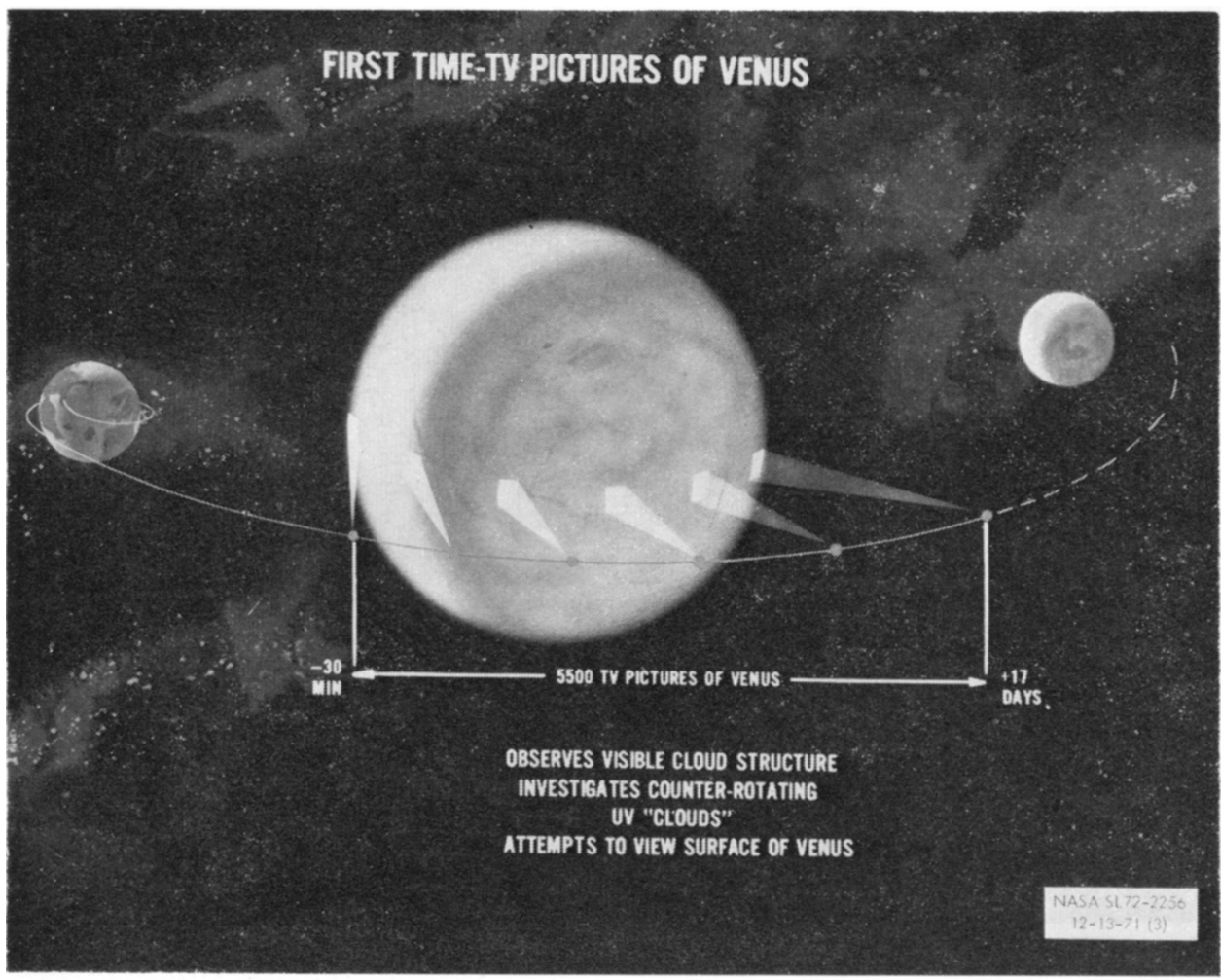

Fig. 5. First time - TV pictures of Venus.

atmosphere of Venus, studied in conjunction with the very tenuous atmosphere of Mars, would yield invaluable insight into the processes and dynamics of Earth's own atmosphere, and aid greatly in our ability to predict the reaction of Earth's atmosphere to the changing conditions on its surface. Exploration of the Venus atmosphere is within our current technological capability, and, in addition to the noted benefits, will provide the data base needed for future surface vehicle design. The program rationale therefore calls for initial lower atmospheric investigations with the Pioneer Venus Probe mission (Figure 6) and more extensive environmental and upper atmosphere studies with Pioneer Venus Orbiter. These will be followed by more detailed atmospheric studies, including a buoyant station to obtain data on atmospheric dynamics.

Mapping the Venusian surface will require the use of radar. Earth-based observations from the large radar telescope near Arecibo, Puerto Rico, will provide surface resolution to about $2 \mathrm{~km}$ over a portion of the planet in the 1975-1980 time period; to obtain resolution to the level of the Mariner 9 Mars maps, will require the use of an orbiting spacecraft with a fully focussed synthetic aperture radar. This is planned for the 1983 time frame. 


\section{VENUS MULTIPLE PROBES}

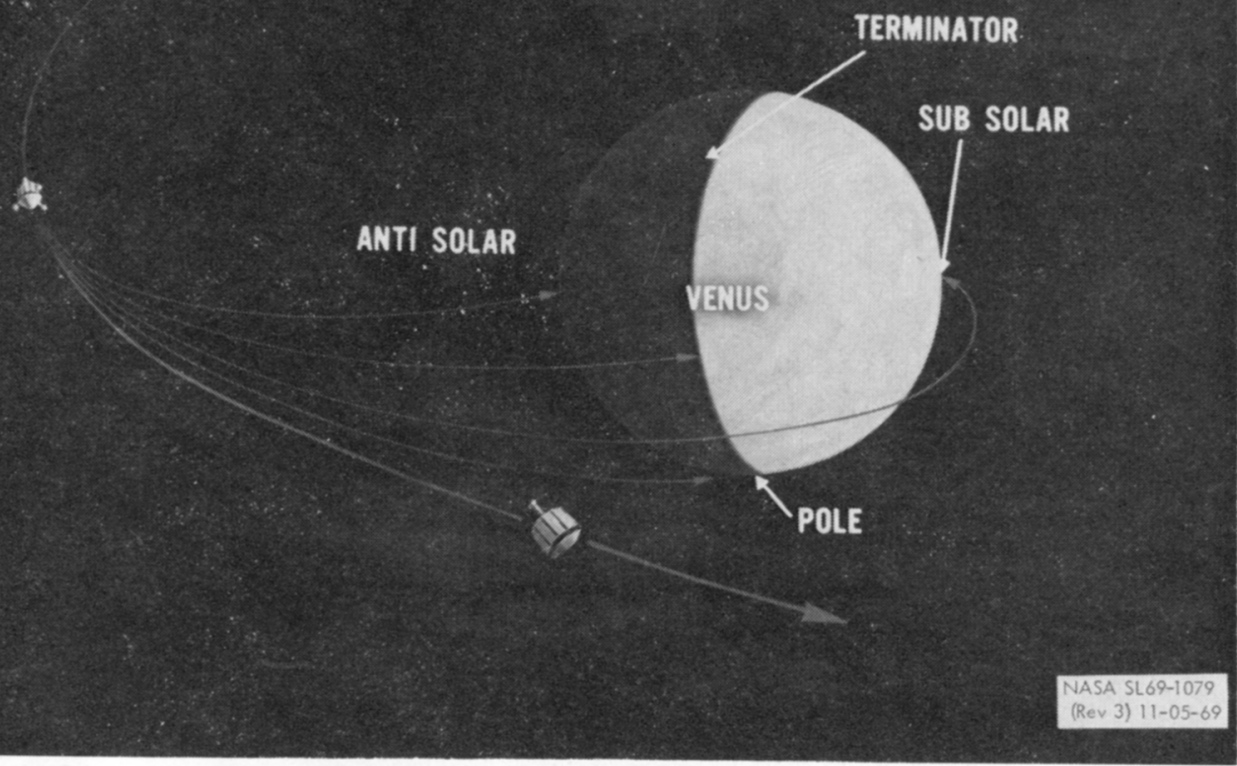

Fig. 6. Pioneer Venus probe mission.

The combination of high resolution surface maps and detailed environmental data are necessary before rational planning for a soft landing mission can commence. Hence no landing on Venus is comtemplated prior to 1989.

\subsection{MARS}

The combination of the low transfer energies required and relatively benign environment allows us to push the exploration of Mars at a much greater pace than any other target. The Mariner 9 mission (Figure 7) has provided us with excellent photographs of the entire Martian surface, as dramatically depicted in Figures 8 and 9, and the Viking mission in 1975 (Figure 10) will provide in situ surface sample analysis and preliminary biological information.

The continuing exploration of Mars now planned includes further Viking Landings in 1979, perhaps with the addition of some roving capability. Whether these missions would be biologically or geologically oriented will depend on the results of the 1975 mission.

Beyond Viking, the next logical step, based on our lunar experience would be the return to Earth of a sample of the Martian surface. This is within the capability of our existing technology and is planned for 1984 . 


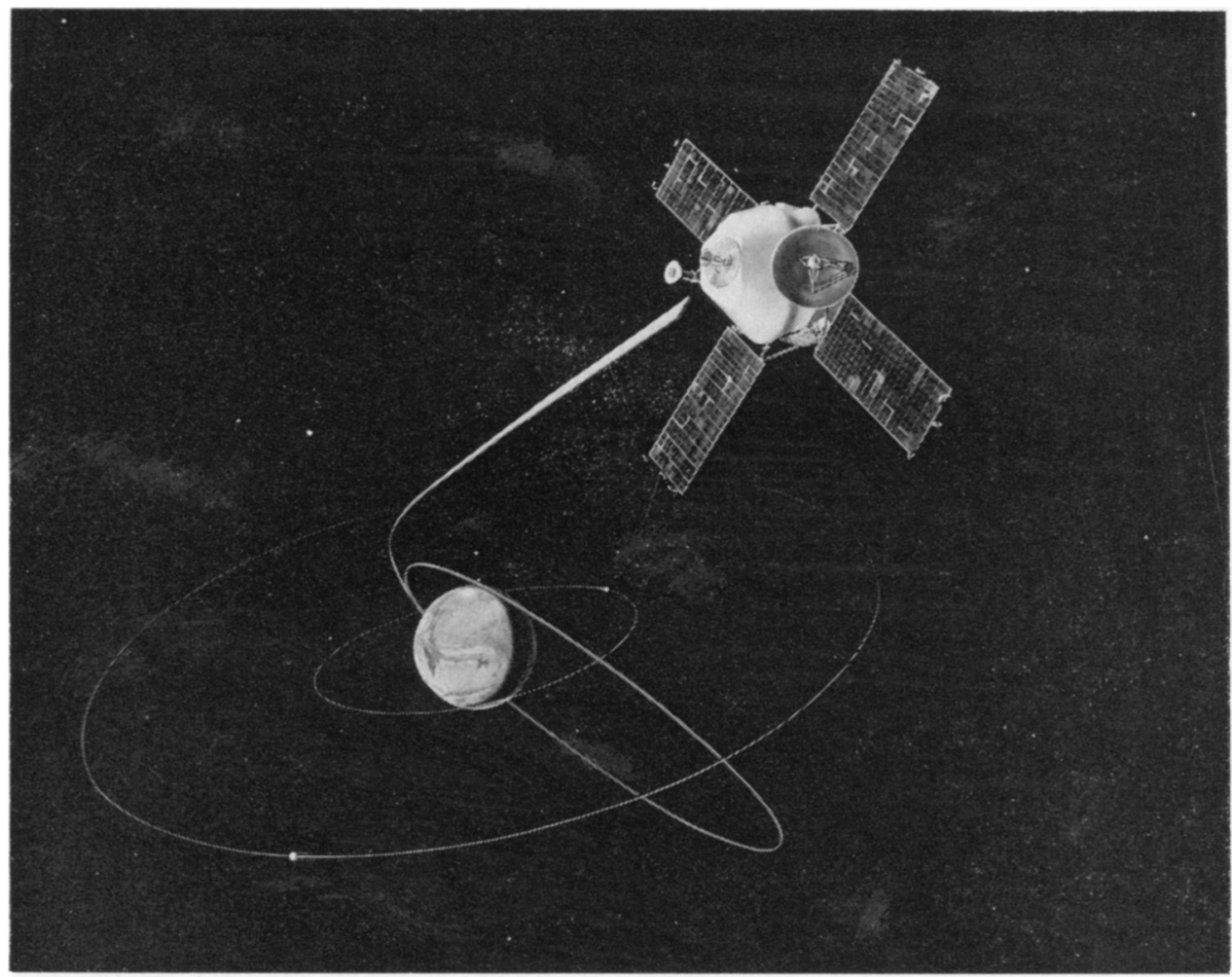

Fig. 7. Mariner 9 mission to Mars.

Also within the scope of existing technology is the landing on and sample return from one of the Martian satellites. However, in the context of the balanced program, this mission is given relatively low priority at the present time and is not contemplated until the 1990 time frame.

\subsection{THE OUTER PLANETS}

There is keen scientific interest in the outer planets and their satellites. The major planets, totally different from the terrestrial planets in size, composition, rotational speed, and other physical characteristics display many anomalous properties which could contain clues to solar system formation processes. The satellites on the other hand have many properties similar to terrestrial planets, and atmospheres have been detected on at least two (Titan and Ganymede). The investigation and understanding of the phenomena of the outer planets ranks high on the priority list of the scientific community.

Until recently, however, the outer planets were beyond our technological capability. Only Jupiter falls within the capability of the Atlas/Centaur, and then only marginally. Further, the distance from the Sun makes the use of solar power impractical, and no other energy source was available. The latter constraint was removed with the develop- 


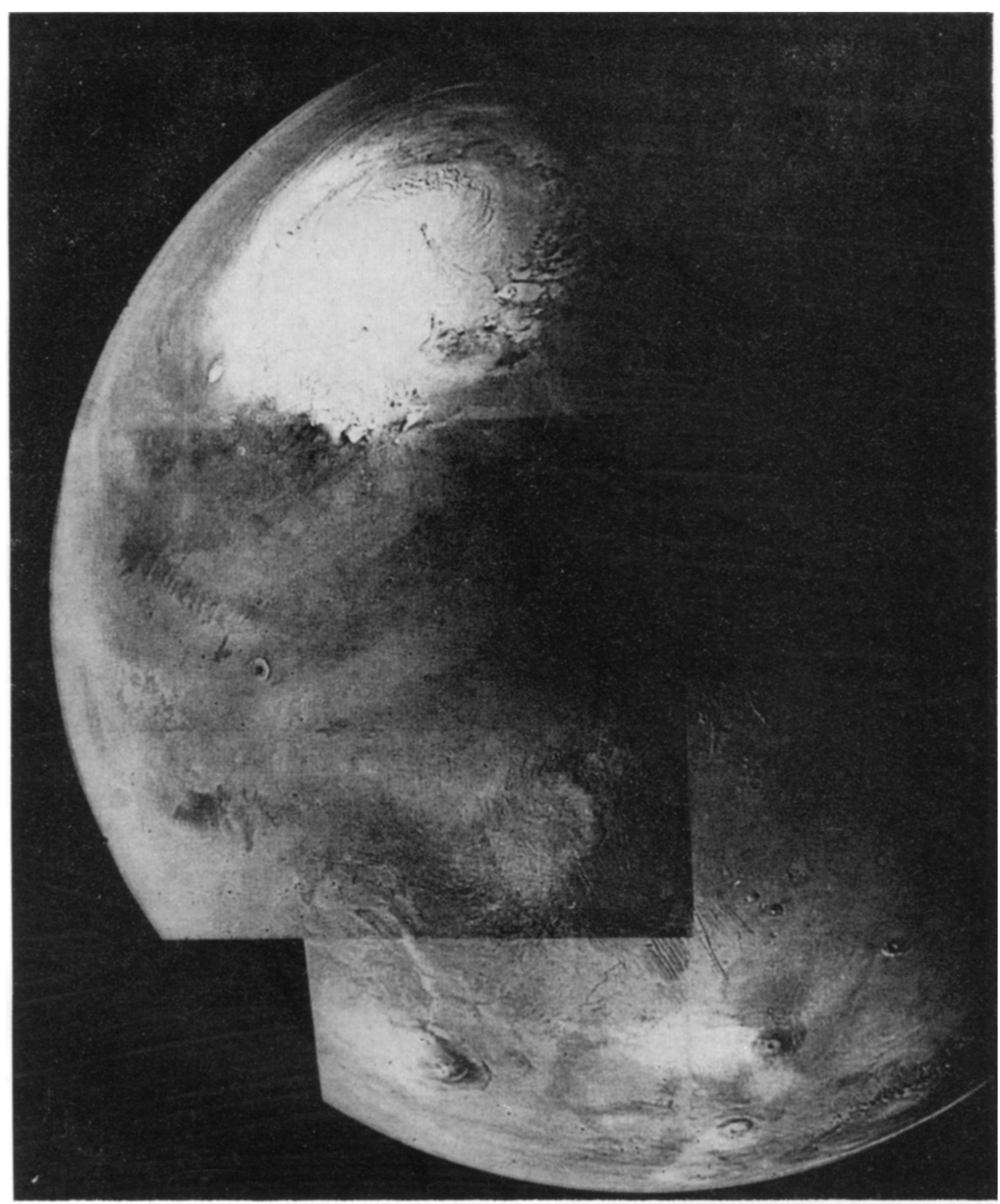

Fig. 8. Mariner 9-global view of Mars.

ment of RTG power supplies, and initial exploration of the outer planets was started with the Pioneer 10 and 11 missions launched in 1972 and 1973. The primary purpose of these spacecraft is to characterize the Jovian magnetosphere by completing the missions shown in Figure 11. This information will be obtained when the spacecraft encounter Jupiter in December 1973 and December 1974, and will provide the data base for future trajectory and spacecraft design constraints. 


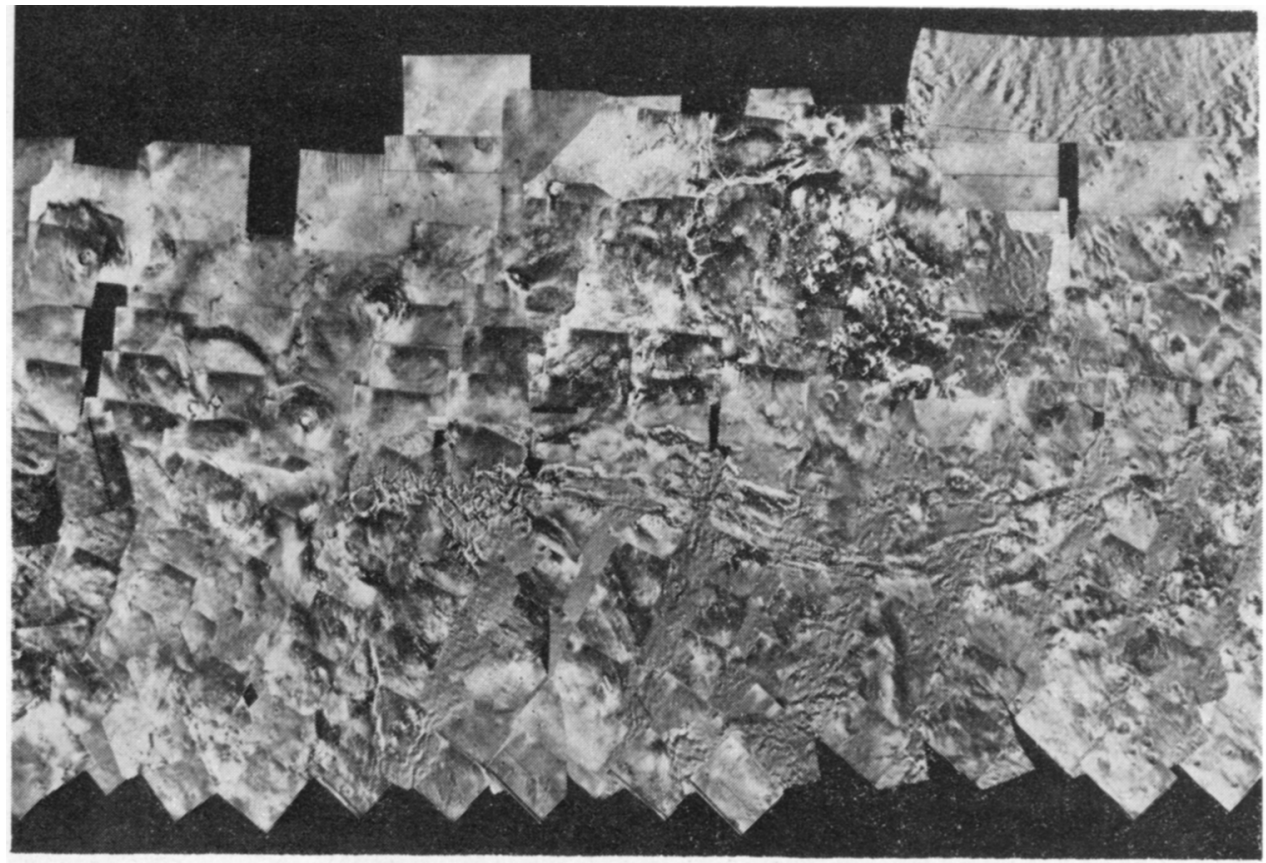

Fig. 9. Mariner 9-photomosaic of portion of Mars equatorial region.

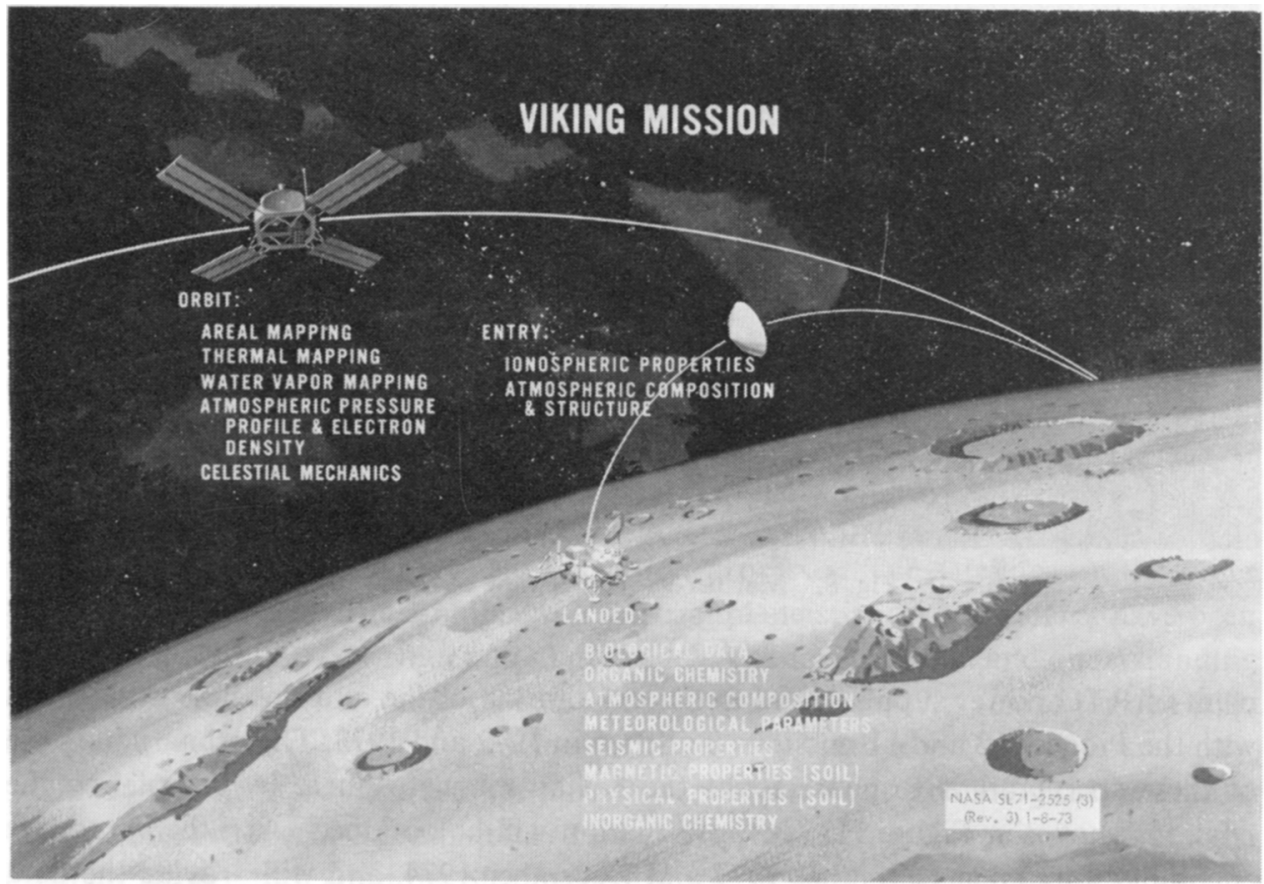

Fig. 10. Viking mission to Mars. 


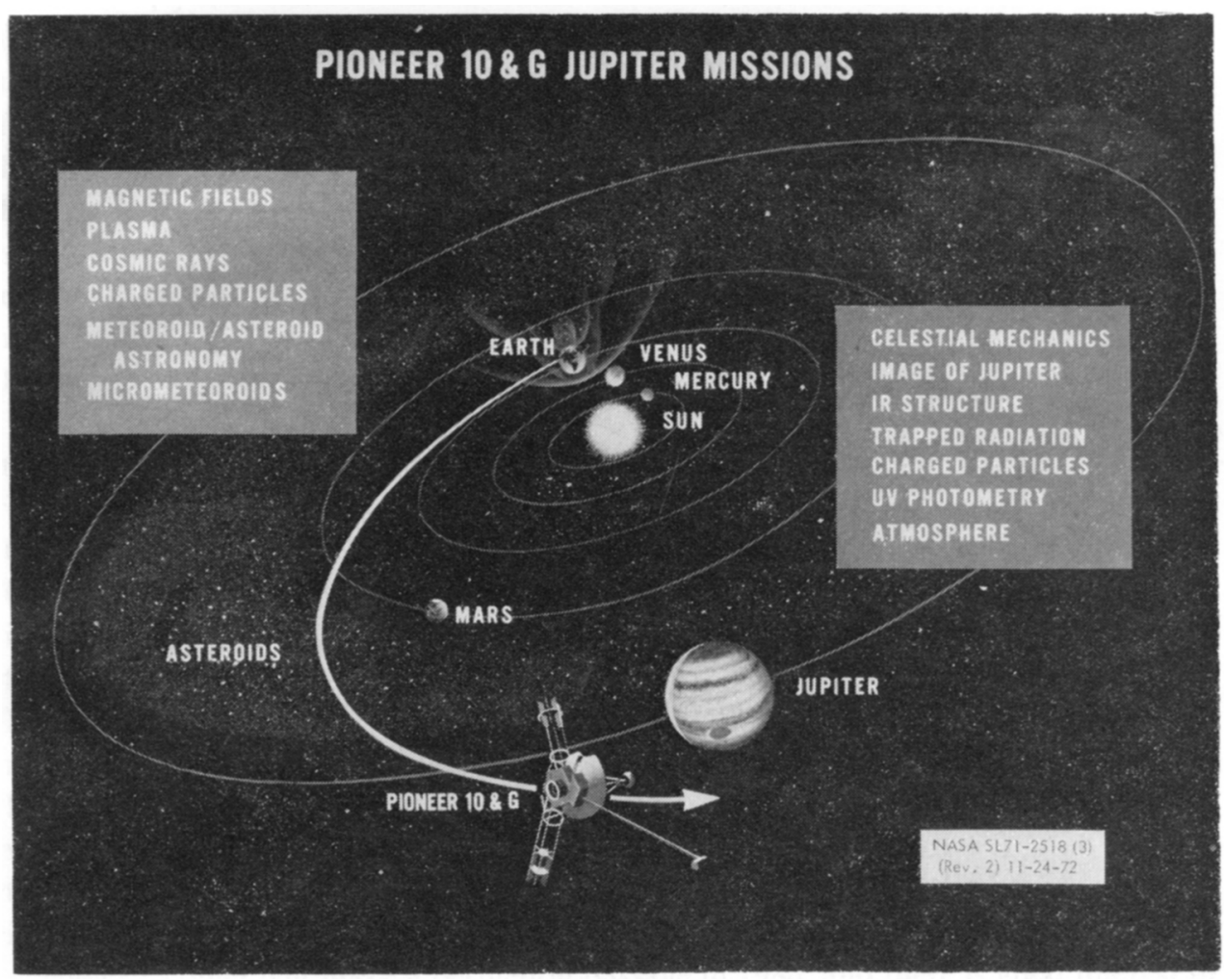

Fig. 11. Pioneer 10 and 11 Jupiter missions.

The launch vehicle capability constraint will be considerably alleviated with the introduction of the Titan/Centaur, and by the unique alignment of the outer planets in the late 70's and early 80's which allow Jupiter and then Saturn to be used as stepping stones to the more distant targets.

Detailed investigations of the outer planets will be pursued along two parallel paths, one emphasizing planetological studies with sophisticated flyby and orbiting spacecraft, and the other emphasizing atmospheric investigations with simpler flyby spacecraft delivering atmospheric probes.

The planetological program will commence with the Mariner Jupiter/Saturn mission in 1977. The objectives of this mission are depicted in Figure 12. The mission will provide close-up imaging in all spectral bands of Jupiter, Saturn, Saturn's Rings, and Titan, and will perform a variety of other science experiments.

In 1979, essentially the same spacecraft will use a Jupiter gravity assist to obtain similar data at Uranus. (Because of spacecraft life time limitations, it is not felt that Neptune could be reached on this mission, although energy-wise, it could be reached using a gravity assist at Uranus.) Following this mission, more detailed investigation of Jupiter is planned with orbiter missions in 1981 and 1982. These missions would provide detailed maps of the planet and its environment and multiple flyby encounters 


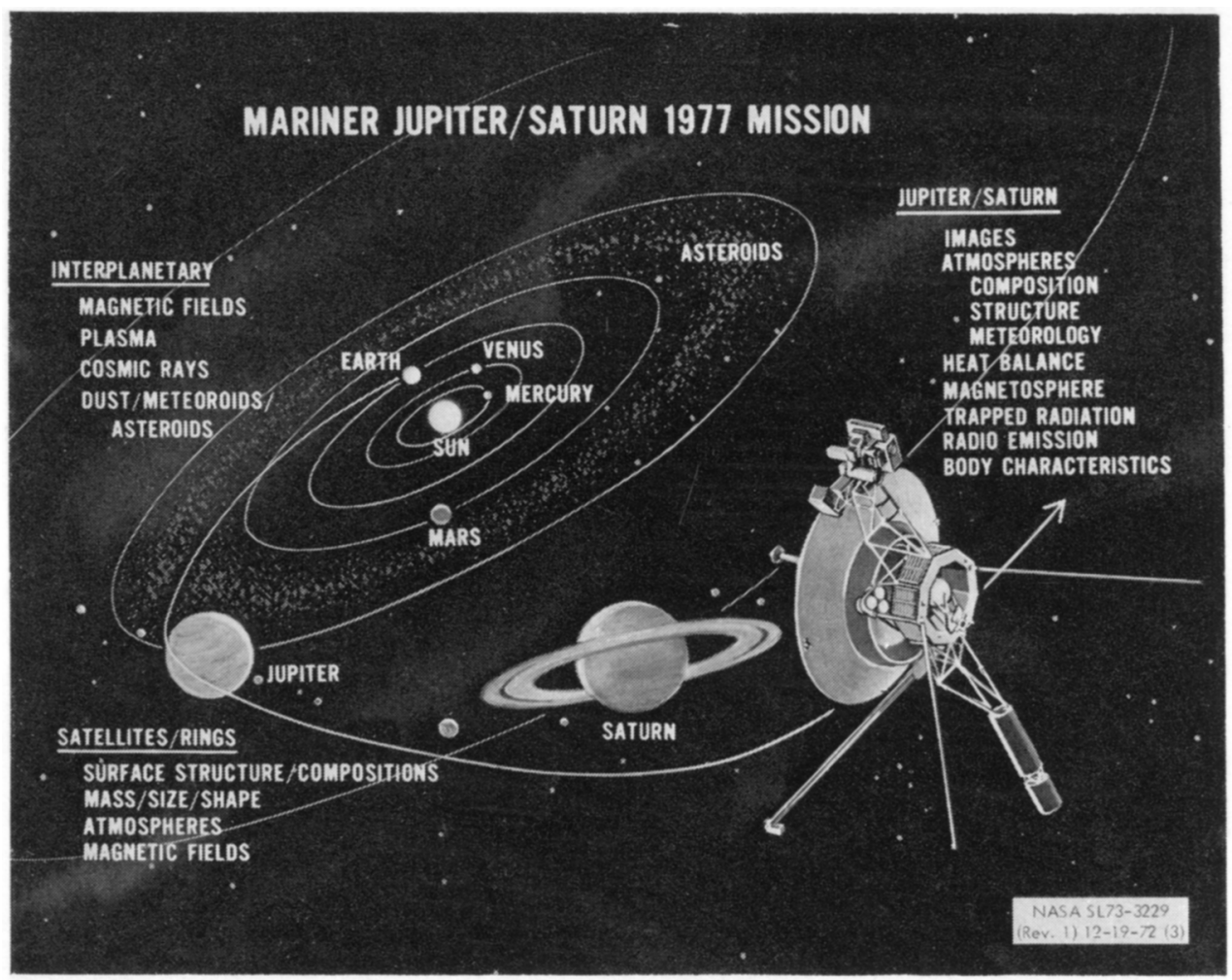

Fig. 12. Mariner Jupiter/Saturn 1977 mission.

with each of the Galilean satellites. By 1985, the introduction of solar-electric capability should permit the orbiting of Saturn, and bring Neptune within reach using a Uranus gravity assist. The latter is planned by 1986 . Finally, it is contemplated that the exploration of the outer planets would enter the lander stage, but this requires propulsion technology beyond anything currently planned. A Ganymede Lander is indicated in the 1990-1991 time frame primarily to serve as a technology driver.

Atmospheric investigation of the outer planets is less demanding, energy-wise, than the planetological exploration because of the generally lighter spacecraft required, and can proceed at a more accelerated pace. Atmospheric probes, derived from the Pioneer Venus technology, would be sent to Saturn in 1979 and to Uranus in 1980. Saturn and Uranus are selected for initial investigation rather than Jupiter because the probe technology requirements are considerably less severe. The third probe of this set will be launched with target selection to be performed en route. This probe could be targeted to Saturn, to Uranus, or possibly to Titan, depending on the status of the earlier probe missions. A Jupiter probe is then contemplated in 1984.

\subsection{COMETS AND ASTEROIDS}

There is growing interest in comet and asteroid missions, based on the supposition 
that they are composed of primordial matter essentially unchanged from the time of their composition. The small bodies thus may hold the best clues on solar system formation processes, and there is growing interest in their early investigation. This is hampered by two considerations. First, only limited information can be obtained from flybys of these objects, particularly asteroids. The very short encounter times severely restrict data acquisition, and their size and the uncertainty in their ephemerides make the problem of navigation for close encounters a difficult one. Second, rendez-vous using chemical propulsion systems is prohibitively expensive energy-wise because of the absence of any significant assistance from local gravity fields.

For many comets and asteroids these limitations can be removed with the introduction of solar-electric propulsion capability, and the mission model envisions a rendezvous with the comet Encke during its 1984 perihelion passage. This would be preceded by a precursor mission during its 1980 perihelion passage which would provide a test bed for cometary science. This precursor could also provide the test flight for SEP, in which case both the coma and tail would be traversed; however, it is possible that fiscal constraints will not permit this. Consequently, a ballistic precursor mission to Encke in 1980 is being studied, with either a single or a dual spacecraft launch. In the single launch mode, the spacecraft would be targeted for the coma; in the dual launch case, the second spacecraft would traverse the tail region. On both the rendezvous and flyby missions initial flybys of asteroids would also be accomplished, leading to an asteroid rendez-vous mission in 1986, and docking and sample return sometime in the 1990's.

Halley's comet will make a perihelion passage in 1986, and there is great interest in visiting this most famous body. Halley's orbit is such that no existing or contemplated propulsion system is capable of providing rendez-vous; however, interest is such that even a very fast ballistic fly through $\left(55 \mathrm{~km} \mathrm{~s}^{-1}\right)$ has strong support. Therefore, a Halley's flyby mission is also being planned to be launched in 1985 .

It is considered that the mission model presented here represents a realistic and well balanced program for solar system exploration, based on the fiscal and technological constraints as we presently understand them. The model, however, is readily subject to change as our understanding of the programmatic environment changes.

\section{DISCUSSION}

Gorgolewski: Do you intend to fly radio astronomy experiments to the outer planets?

Brunk: Yes, there is a radio astronomy experiment being considered for the Mariner-JupiterSaturn mission. Such experiments may also be flown on later missions. All programs will also make maximum scientific use of the spacecraft transmitter for radio occultation experiments, etc.

Dollfus: What are the NASA programs for asteroids and comets?

Brunk: There are several missions being studied, such as a Comet Encke mission and one to Halley's Comet. Also under study are multiple flyby missions which would include one or more asteroids and one or more comets. 